\title{
The Analysis of Sensitive Materials Using EBSD: The Importance of Beam Conditions and Detector Sensitivity
}

\author{
Pat Trimby ${ }^{1 *}$, Angus Bewick ${ }^{1}$, Daniel Abou-Ras ${ }^{2}$, Pietro Caprioglio $^{3}$, Dieter Neher $^{3}$ and Laura Otter ${ }^{4}$ \\ 1. Oxford Instruments Nanoanalysis, Halifax Road, High Wycombe, UK. \\ 2. Helmholtz-Zentrum Berlin für Materialien und Energie GmbH, Berlin, Germany. \\ 3. Institut für Physik und Astronomie, Universität Potsdam, Potsdam-Golm, Germany. \\ 4. Department of Earth \& Planetary Science, Macquarie University, Sydney, Australia. \\ * Corresponding author: pat.trimby@oxinst.com
}

Materials that are sensitive to electron beam microanalysis are typically very challenging to analyze using electron backscatter diffraction (EBSD). However, such materials play an important role in many key application fields, notably in the energy industry (e.g. halide perovskite-based solar cells) and in the biogeosciences (e.g. shell structures and bone/teeth) [1, 2]. The successful analysis of such materials requires carefully selected sampling strategies [e.g. 3], electron beam parameters and, most importantly of all, a high EBSD detector sensitivity.

There remains significant confusion within the literature about the best approach to analyzing beamsensitive samples as well as a lack of any standardized approach to measuring EBSD detector sensitivity. Frequently, published papers fail to provide relevant analytical details (e.g. [4]), making an assessment of sample stability and beam-sensitivity extremely challenging. In this presentation we look in detail at the key parameters: we consider the impact of electron beam energy (acceleration voltage), electron beam current, electron dose (both on the sample and the EBSD detector) and EBSD detector design. We find that 2 electron beam parameters are paramount: the accumulated electron dose on the surface of the sample (i.e. beam current $x$ exposure time / unit area) and the beam energy (accelerating voltage). For beam sensitive materials, maintaining a low accumulated dose is essential, and we examine the impact of detector technology and improvements in detector sensitivity in this respect. In particular, we look at how fiber optic coupling between the detector scintillator and a customized complementary metal-oxide semiconductor (CMOS) sensor provides a significant increase in detector sensitivity.

The relationship between beam energy and the successful analysis of beam-sensitive samples is complex. In general, a lower beam energy helps to reduce sample damage but this comes at a cost of speed, not least due to the lower efficiency of the phosphor scintillator in converting the backscattered electron signal to light. An accelerating voltage of between 8 and $15 \mathrm{keV}$ is ideal for most samples, with the lower energy also providing a benefit in the spatial resolution of the EBSD results (and any associated energy dispersive X-ray spectrometry (EDS) measurements).

The use of a high-efficiency, fiber-optic coupled CMOS sensor enables the effective analysis of samples that have previously been difficult or impossible to characterize using EBSD. In this paper we present examples from both biomaterials, such as aragonitic clam shells, and from organic and inorganic perovskite thin films, as shown in figure 1. We also examine the potential impact of energy-filtered direct electron detection (EF-DeD $[5,6]$ ) in the use of EBSD to study sensitive materials. EF-DeD offers the potential for greater detector sensitivity than is possible with even the most efficient, opticallycoupled system, enabling successful analyses at the lowest possible accumulated doses. 
The results suggest that the introduction of new detector technologies, either using fiber optic coupled CMOS sensors or direct electron detection, is significantly enhancing our capability to examine beam sensitive materials using EBSD. This is likely to have a profound impact on the analysis of organic samples and biomaterials in the coming years.

\section{References:}

[1] NJ Jeon et al., Nature 517 (2015), p. 476.

[2] L Otter et al., Biogeosciences (2019). https://doi.org/10.5194/bg-2018-469

[3] K Abbasi et al., Microscopy \& Microanalysis 24 (2018), p. 420.

[4] C Jiang and P Zhang, J. Appl. Phys 123 (2018), p. 083105.

[5] US Patent US8890065B2: Apparatus and method for performing microdiffraction analysis.

[6] S Vespucci et al., Physical Review B 92 (2015), p. 205301.
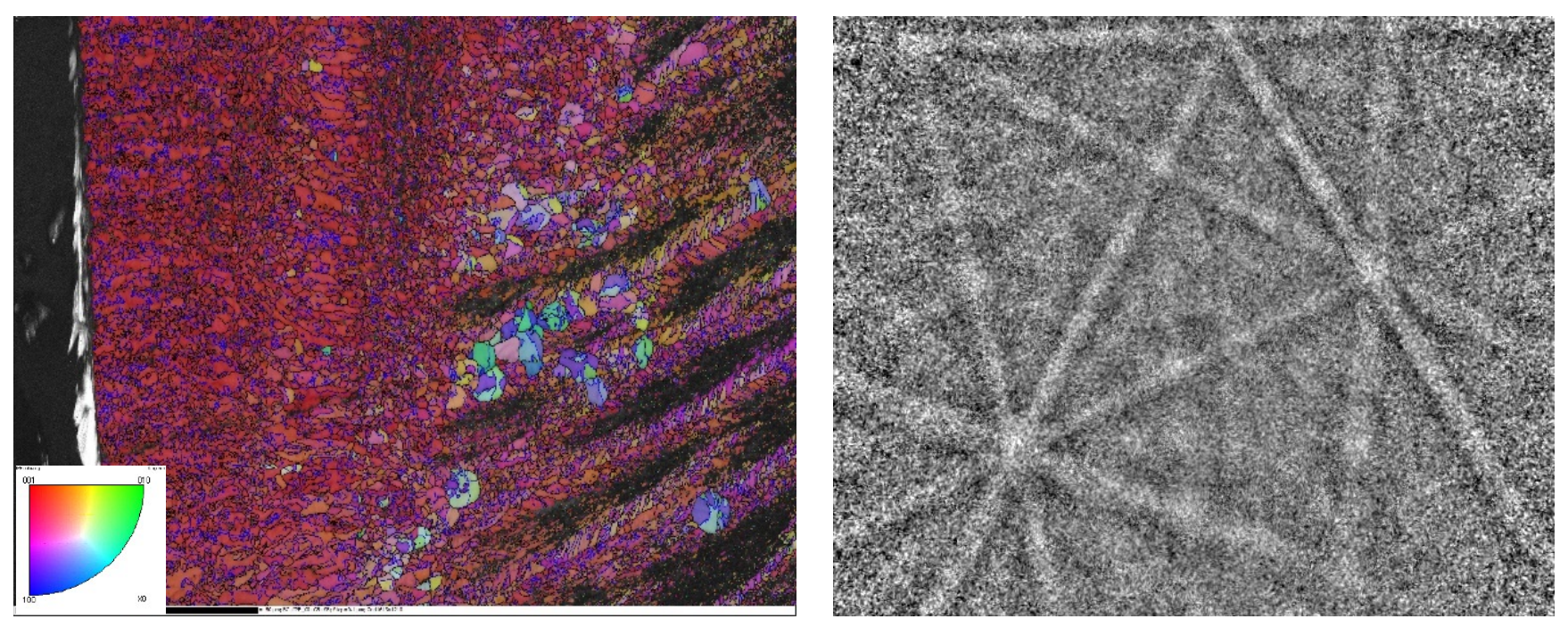

Figure 1. Left: Orientation map of a clam sample (Katelysia rhytiphora) analysed at $100 \mathrm{~nm}$ step size using $15 \mathrm{kV}$ with a dose per pixel of $50 \mathrm{nAms}$. Inset - colour scheme for aragonite orientation (IPF-X direction). Field of view is 161 um across. Right: Example EBSD pattern from an organic halide perovskite thin film (MAPI type) collected using $12 \mathrm{kV}$ with a dose of $29 \mathrm{nAms}$. 